\title{
WHAT CAN WE LEARN FROM THE RISING LIGHT CURVES OF RADIOACTIVELY POWERED SUPERNOVAE?
}

\author{
AnThony L. Piro ${ }^{1}$ and Ehud NAKAR ${ }^{2}$ \\ ${ }^{1}$ Theoretical Astrophysics, California Institute of Technology, 1200 E California Blvd., M/C 350-17, Pasadena, CA 91125, USA; piro@ caltech.edu \\ ${ }^{2}$ Raymond and Beverly Sackler School of Physics and Astronomy, Tel Aviv University, Tel Aviv 69978, Israel \\ Received 2012 October 8; accepted 2013 April 8; published 2013 May 6
}

\begin{abstract}
The light curve of the explosion of a star with a radius $\lesssim 10-100 R_{\odot}$ is powered mostly by radioactive decay. Observationally, such events are dominated by hydrogen-deficient progenitors and classified as Type I supernovae (SNe I), i.e., white dwarf thermonuclear explosions (Type Ia), and core collapses of hydrogen-stripped massive stars (Type $\mathrm{Ib} / \mathrm{c}$ ). Current transient surveys are finding SNe I in increasing numbers and at earlier times, allowing their early emission to be studied in unprecedented detail. Motivated by these developments, we summarize the physics that produces their rising light curves and discuss ways in which observations can be utilized to study these exploding stars. The early radioactive-powered light curves probe the shallowest deposits of ${ }^{56} \mathrm{Ni}$. If the amount of ${ }^{56} \mathrm{Ni}$ mixing in the outermost layers of the star can be deduced, then it places important constraints on the progenitor and properties of the explosive burning. In practice, we find that it is difficult to determine the level of mixing because it is hard to disentangle whether the explosion occurred recently and one is seeing radioactive heating near the surface or whether the explosion began in the past and the radioactive heating is deeper in the ejecta. In the latter case, there is a "dark phase" between the moment of explosion and the first observed light emitted once the shallowest layers of ${ }^{56} \mathrm{Ni}$ are exposed. Because of this, simply extrapolating a light curve from radioactive heating back in time is not a reliable method for estimating the explosion time. The best solution is to directly identify the moment of explosion, either through observing shock breakout (in X-ray/UV) or the cooling of the shock-heated surface (in UV/optical), so that the depth being probed by the rising light curve is known. However, since this is typically not available, we identify and discuss a number of other diagnostics that are helpful for deciphering how recently an explosion occurred. As an example, we apply these arguments to the recent SN Ic PTF 10vgv. We demonstrate that just a single measurement of the photospheric velocity and temperature during the rise places interesting constraints on its explosion time, radius, and level of ${ }^{56} \mathrm{Ni}$ mixing.
\end{abstract}

Key words: hydrodynamics - shock waves - supernovae: general

Online-only material: color figures

\section{INTRODUCTION}

A typical supernova (SN) light curve is powered by a combination of two sources: (1) the energy deposited by the SN shock and (2) the radioactive decay of ${ }^{56} \mathrm{Ni}$ that was synthesized during the explosion. ${ }^{3}$ The first light from shock breakout and the following early emission (for the first minutes to days) is dominated by the shock-deposited energy. The very late emission, after the radiation can efficiently diffuse through the entire ejecta ( $\gtrsim 100$ days), is dominated by radioactive decay. The relative influence of these power sources during the time in between depends on two main factors: (1) the progenitor radius $R_{*}$ and (2) the amount of ${ }^{56} \mathrm{Ni}$. The reason for this is that the bolometric luminosity from shock heating increases linearly with the progenitor radius (as we discuss in Section 2.2), while the peak of the radioactively powered luminosity is roughly linear with the total mass of ${ }^{56} \mathrm{Ni}$ (Arnett 1979).

Core-collapse SNe typically synthesize $\sim 0.01-0.1 M_{\odot}$ of ${ }^{56} \mathrm{Ni}$, and the progenitor radii range between $\sim 1$ and $1000 R_{\odot}$. In more extended progenitors, the main $\mathrm{SN}$ event is dominated by shock heating, while in more compact ones it is dominated by radioactive power. The most common SNe II-P are explosions of red supergiants with $R_{*} \sim 500 R_{\odot}$. They exhibit an extended

\footnotetext{
3 Here, we ignore more exotic energy sources such as interaction of ejecta with circumsupernova matter (such as in Type IIn SNe) and spin-down of a rapidly rotating magnetar (Kasen \& Bildsten 2010; Woosley 2010).
}

plateau phase for about a hundred days, which is powered by the cooling of shock-heated material. The progenitors of the rare 1987A-like Type II supernovae (SNe II) are blue supergiants with $R_{*} \sim 50 R_{\odot}$ (Woosley 1988; Kleiser et al. 2011), and their light curves are dominated by radioactive decay starting a week after the explosion. Similarly, SNe IIb with compact progenitors $\left(R_{*} \lesssim 10 R_{\odot}\right)$ show evidence of both shock-heated material and a separate radioactive peak. The progenitors of $\mathrm{SNe} \mathrm{Ib} / \mathrm{c}$ are massive stars that were stripped of their hydrogen envelope and are also fairly compact with $R_{*} \lesssim 10 R_{\odot}$. Indeed $\mathrm{SNe} \mathrm{Ib} / \mathrm{c}$ are dominated by radioactive power starting a few days (or earlier) after the explosion. Finally, for the compact white dwarf (WD) progenitors of SNe Ia with $R_{*} \lesssim 0.01 R_{\odot}$, the shock-heating light curves are so dim that they have never been observed, and our knowledge of these events is only possible due to the synthesis of $\sim 0.5 M_{\odot}$ of ${ }^{56} \mathrm{Ni}$.

Since the physics that governs the light curves of most $\mathrm{SNe}$ with $R_{*} \lesssim 50 R_{\odot}$ has many similarities, a single theoretical framework should roughly describe their main qualitative features. Their emission during the first few days is especially exciting because it probes the shallowest layers of the progenitor. This can teach us about the exploding star's radius, and constrain the surface composition and velocity/density gradients that reflect details of the explosive burning. The shock-heating contribution in SNe I has been well studied in the literature, both with semi-analytic models (Nakar \& Sari 2010; Rabinak \& Waxman 2011) and with detailed radiative transfer simulations 
(Dessart et al. 2011). The rising light curve from radioactive heating has been explored for the cases of SNe Ia (Piro 2012) and to study the impact of ${ }^{56} \mathrm{Ni}$ mixing in $\mathrm{SNe} \mathrm{Ib} / \mathrm{c}$ (Dessart et al. 2012).

Concurrent with these theoretical studies, transient surveys like with the Katzman Automatic Imaging Telescope (Filippenko et al. 2001), and by the Palomar Transient Factory (PTF; Rau et al. 2009; Law et al. 2009) and the Panoramic Survey Telescope and Rapid Response System (Kaiser et al. 2002) are finding increasing numbers of these events, especially at early times. Best known among these is SN 2011fe, the closest SNe Ia in the last 25 years (Nugent et al. 2011; Bloom et al. 2012). Other SNe Ia reported within the last year with early data include SN 2009ig (Foley et al. 2012), SN 2010jn (Hachinger et al. 2013), and SN 2012cg (Silverman et al. 2012). SNe Ib/c have also been increasingly well studied in the optical at early times, as summarized by Drout et al. (2011). Other particular recent events include SN 2008D (Soderberg et al. 2008; Modjaz et al. 2009) and PTF 12gzk (Ben-Ami et al. 2012). Motivated by these exciting developments, we ask: what can and what cannot be learned from the early optical light curve of radioactively powered SNe? Being more abundant, we focus on parameters that are typical to SNe I, although most of our conclusions can also be applied to subclasses of SNe II that are dominated by radioactive heating.

In our study, we first consider instances where only a photometric light curve is available, as is often the case at early times. One of the main issues is how much we can infer about ${ }^{56} \mathrm{Ni}$ mixing in the outermost layers of the star with this limited information. Our main conclusion is that it is hard to determine the mixing of ${ }^{56} \mathrm{Ni}$ based on the light curve alone without additional information. The reason is that the light curve can provide an estimate for the total mass of ${ }^{56} \mathrm{Ni}$ that is exposed to the observer at any given time, but it cannot provide a good handle on the fractional mass of ${ }^{56} \mathrm{Ni}$ with respect to the total exposed mass (which can be determined if the time of explosion is well constrained, e.g., by detection of the shock breakout or the cooling envelope phase). Putting it differently, there is a degeneracy in the light curve between a recent explosion with a high fraction of ${ }^{56} \mathrm{Ni}$ in the outermost layers and an older explosion where ${ }^{56} \mathrm{Ni}$ resides only in deep material. In the latter case, the SN has a "dark phase" that can persist for up to a few days after the explosion. During this time, no ${ }^{56} \mathrm{Ni}$ is exposed and its shock-heated cooling emission is often too faint for detection even when deep observations exist. For this reason, a simple extrapolation of the light curve to early times cannot reliably constrain the time of explosion.

We then consider what additional information can be extracted by using limited spectral data that provide the photosphere temperature and velocity. We show that if detailed color evolution, or if one or, even better, two spectra (possibly of low signal to noise) are available during the rise, then the degeneracy between explosion time and ${ }^{56} \mathrm{Ni}$ depth can be at least partially alleviated. Key among our results is Equation (17), which allows one to estimate a lower limit on the time of explosion using merely a single simultaneous measurement of the bolometric luminosity, temperature, and photospheric velocity. The methods we discuss are complementary to detailed spectral studies that probe element mixing based on their absorption and emission features in the rare cases where early high signal to noise spectra are available (e.g., Sauer et al. 2006; Parrent et al. 2012).

In the following, we begin in Section 2 by discussing each of the ingredients that shape the electromagnetic emission

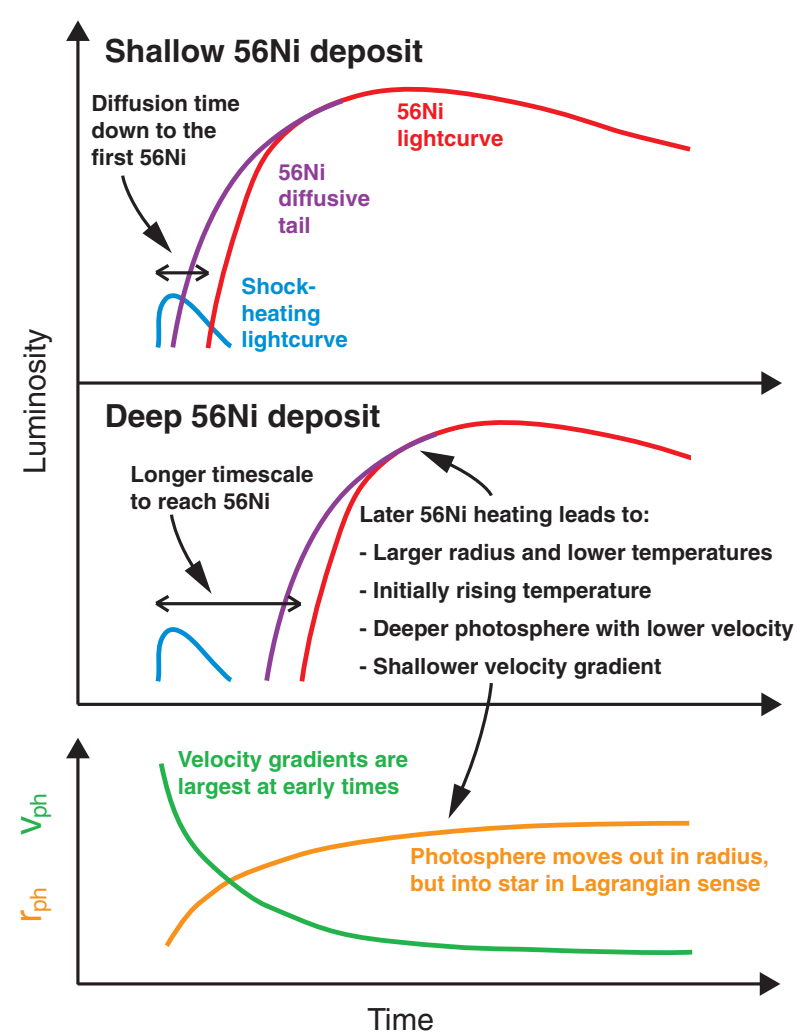

Figure 1. Schematic diagram showing the early light curves for SNe I and how they relate to the photospheric radius and velocity. The top and middle panels demonstrate how the relative positions of the shock-heating light curve (blue curves, discussed in Section 2.2), diffusive tail light curve (purple curves, discussed in Section 2.4), and the ${ }^{56} \mathrm{Ni}$ light curve (red curves, discussed in Section 2.3) can differ depending on the depth of the ${ }^{56} \mathrm{Ni}$. The total observed light curve is the sum of these three components. Note that these light curves are meant to replicate the shapes when the luminosity is plotted logarithmically and the time is plotted linearly. When the ${ }^{56} \mathrm{Ni}$ is deposited deeply (in the middle panel) and the shock-heating light curve (blue curve) is below the detection limits, there can be a significant dark phase between the time of explosion and the moment of first detection. In the bottom panel, we show how the photospheric radius (orange curve) and velocity (green curve) evolve with time. Depending on the position of the ${ }^{56} \mathrm{Ni}$ light curve, different photospheric radii, velocities, and velocity gradients will be present when the rising light curve is observed. These provide clues about the depth of the ${ }^{56} \mathrm{Ni}$ and the time of explosion, as summarized in the middle panel and discussed in the text.

(A color version of this figure is available in the online journal.)

starting less than an hour and up to weeks after explosion. This discussion provides a useful general guide for interpreting early observations of radioactively dominated SNe. In Section 3, we investigate a specific SN Ic in some detail (PTF 10vgv) as a test case for applying these arguments and techniques. We conclude in Section 4 with a discussion of our results and a summary of important conclusions that should help facilitate better constraints from future SNe I observations.

\section{EARLY EMISSION FROM TYPE I SUPERNOVAE}

When a hydrogen-poor star explodes as an $\mathrm{SN}$, a few important events occur in the moments before and after the first optical emission is seen. In this section, we summarize the main properties of each of these events, their observational consequences, and how detections of some or all of these events can be used to put constraints on the properties of the exploding star. To guide the discussion, we will be referring to the diagram in Figure 1, which shows the time-dependent luminosity components, photospheric radius, and velocity. 


\subsection{Shock Breakout}

Just prior to emission, a shock is traveling through the envelope. This heats and accelerates the material, unbinding it from the star. A radiation-dominated shock accelerates in the decreasing density of the outer edge of the star with a shock velocity that scales with the density as $v_{s} \propto \rho^{-\beta}$ where $\beta \approx 0.19$ (Sakurai 1960). The shock continues to shallower regions until the optical depth falls to $\tau \approx c / v_{s}$, where $c$ is the light speed. At this point, the photons are no longer trapped; they stream away and the shock dies. This is what is typically referred to as the "shock breakout" UV/X-ray flash, and it has been frequently studied because of its strong dependence on the radius (which determines both the energy budget of the shock and the timescale of the emission), allowing the progenitor star to be studied from its detection (Colgate 1974; Falk 1978; Klein \& Chevalier 1978).

Recently, it was realized that the radiation behind the shock falls out of thermal equilibrium for small radii hydrogenstripped massive stars with high shock velocities, and that the breakout will be in X-rays (Katz et al. 2010; Nakar \& Sari 2010). In SNe Ia, the shock achieves relativistic velocity, and its breakout emission is in the MeV range (Nakar \& Sari 2012). In any case, although shock breakout is the first indication of the explosion, its impact for SNe I in the optical/UV bands is negligible. For this reason, it is not discussed further in this paper and not plotted in Figure 1.

\subsection{Shock-heated Cooling Light Curve}

Immediately after shock breakout, the observed radiation is out of thermal equilibrium until roughly the time when the gas doubles its radius at $t \approx R_{*} / v_{f}$ (Nakar \& Sari 2010), where $v_{f} \approx 2 v_{s}$ is the final velocity of the material (Matzner \& McKee 1999), which is achieved within minutes or less. The observed photons then gain thermal equilibrium and the expansion enters its spherical homologous phase (Chevalier 1992; Piro et al. 2010; Nakar \& Sari 2010; Rabinak \& Waxman 2011). As the material that has been heated by the shock expands, a thermal diffusion wave begins backing its way through the ejecta. Above the depth of the diffusion wave, material cools via photon diffusion. Below this depth, material evolves adiabatically. For each fluid element, there is then a competition between adiabatic cooling and diffusive cooling that controls the energy density of that material at the moment its photons begin streaming out of the star. This determines the observational signature of shockheated cooling, leading to a bolometric luminosity (Nakar \& Sari 2010) $)^{4}$

$$
L \approx 2 \times 10^{41} \frac{E_{51}^{0.91} R_{1}}{\kappa_{0.2}^{0.82} M_{1}^{0.73}}\left(\frac{t}{1 \mathrm{hr}}\right)^{-0.35} \mathrm{erg} \mathrm{s}^{-1}
$$

and an observed (color) temperature of

$$
T_{c} \approx 4 \frac{E_{51}^{0.11} R_{1}^{0.38}}{\kappa_{0.2}^{0.23} M_{1}^{0.11}}\left(\frac{t}{1 \mathrm{hr}}\right)^{-0.61} \mathrm{eV},
$$

where $E=E_{51} 10^{51} \mathrm{erg}$ is the explosion energy, $M=M_{1} M_{\odot}$ is the ejecta mass, $R_{1}=R_{*} / R_{\odot}$, and $\kappa$ is the opacity with $\kappa_{0.2}=\kappa / 0.2 \mathrm{~cm}^{2} \mathrm{~g}^{-1}$ being the canonical value during the cooling phase for fully ionized hydrogen-free gas. For all the scalings in this paper, we assume a polytropic index of $n=3$,

\footnotetext{
4 The luminosity prefactor of Nakar \& Sari (2010) is divided here by a factor
} of 2.5 following the numerical result of Katz et al. (2012).

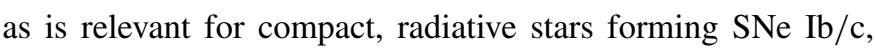
or relativistic, degenerate WDs exploding as SNe Ia. The key result is that the luminosity during the shock-heated cooling is directly proportional to the progenitor radius (as was used to derive radius constraints for SN 2011 fe by Bloom et al. 2012).

In the optical/UV, the luminosity is rising as long it is in the Rayleigh-Jeans tail, resulting in (see Appendix A)

$$
L_{\mathrm{opt} / \mathrm{UV}} \propto t^{1.5}
$$

When optical/UV luminosity is observed to rise more steeply than this (as in PTF 12gzk; Ben-Ami et al. 2012), it indicates that the rise from shock-heated cooling is not being seen, and the time of explosion is not confidently constrained. This phase continues for several hours in a core-collapse SN until $T_{c}$ crosses the UV (at which point the UV flux starts to drop) and reaches the optical band. This is also the point that recombination starts in cases that radioactive decay does not play an important role (as further discussed the next paragraph). In SNe Ia, this phase is terminated after about an hour when the diffusion wave reaches material where the shock was not radiation-dominated and the cooling envelope emission drops significantly (Rabinak et al. 2012).

Once $T_{c}$ drops sufficiently in a core-collapse $\mathrm{SN}$, a recombination wave begins backing its way in from the surface. The temperature drop becomes more gradual, settling at $\approx 5000-8000 \mathrm{~K}$ and the luminosity drop stops or even gently rises (as discussed in Dessart et al. 2011; also see T. Goldfriend et al., in preparation). As far as optical photometry is considered, both the temperature and the luminosity are roughly constant and the SN enters a "plateau phase." This plateau is similar to the one observed in SN II-P (Popov 1993; Kasen \& Woosley 2009), except that for $\mathrm{SNe} I$ it is dimmer and it starts earlier due to the smaller progenitor radius. Setting $T_{c} \approx 0.6 \mathrm{eV}$ as found for the plateau in the SN Ib/c models of Dessart et al. (2011), Equation (2) implies that the optical plateau starts at

$$
t_{p} \approx 20 \frac{E_{51}^{0.18} R_{1}^{0.62}}{\kappa_{0.2}^{0.38} M_{1}^{0.18}} \mathrm{hr},
$$

and together with Equation (1) we find that

$$
L_{p} \approx 7 \times 10^{40} \frac{E_{51}^{0.85} R_{1}^{0.78}}{\kappa_{0.2}^{0.69} M_{1}^{0.67}} \operatorname{erg~s}^{-1}
$$

is roughly the plateau luminosity. ${ }^{5}$

To conclude, the detection of the shock-heated cooling phase in core-collapse $\mathrm{SNe} \mathrm{I}$ is very challenging given its low luminosity and short duration, but the rewards are high. Photometry alone of the rising phase provides tight constraints on the explosion time, and both the optical rising and subsequent plateau phase provide constraints on the progenitor radius (if not overcast by radioactive heating as we discuss next). A convenient method to identify that a rising optical emission is due to shock-heated cooling emission (and not radioactively powered emission) is that the optical emission rises (as predicted by Equation (3)) while the bolometric luminosity drops. This can be seen by having simultaneous UV and optical coverage in the time that $T_{c}$ sits between these two frequency windows.

\footnotetext{
5 Applying the same line of arguments to a red supergiant with $R_{*}=500 R_{\odot}, M=15 M_{\odot}$, and $\kappa=0.34 \mathrm{~cm}^{2} \mathrm{~g}^{-1}$, results in $t_{p} \approx 10$ days and $L_{p} \approx 10^{42} \mathrm{erg} \mathrm{s}^{-1}$, compatible with observations of SNe II-P.
} 


\section{3. ${ }^{56} \mathrm{Ni}$ Shallower than the Diffusion Depth}

If there was no radioactive energy input, this would be the end of the story for the electromagnetic signal from $\mathrm{SNe}$, and most SNe I would be too dim to have ever been detected. But eventually continuous energy release by the radioactive decay of ${ }^{56} \mathrm{Ni}$ starts to dominate the observed luminosity, and this is where their light curves begin rising in earnest. When the thermal diffusion wave reaches the shallowest deposits of ${ }^{56} \mathrm{Ni}$, the energy generation from ${ }^{56} \mathrm{Ni}$ roughly goes directly into the observed bolometric luminosity (as shown with the red curves in Figure 1), so that

$$
L_{56} \approx M_{56} \epsilon,
$$

where the specific heating rate from ${ }^{56} \mathrm{Ni}$ is

$$
\epsilon(t)=\epsilon_{\mathrm{Ni}} e^{-t / t_{\mathrm{Ni}}}+\epsilon_{\mathrm{Co}}\left(e^{-t / t_{\mathrm{Co}}}-e^{-t / t_{\mathrm{Ni}}}\right),
$$

where $\epsilon_{\mathrm{Ni}}=3.9 \times 10^{10} \mathrm{erg} \mathrm{g}^{-1} \mathrm{~s}^{-1}, t_{\mathrm{Ni}}=8.76$ days, $\epsilon_{\mathrm{Co}}=$ $7.0 \times 10^{9} \mathrm{erg} \mathrm{g}^{-1} \mathrm{~s}^{-1}$, and $t_{\mathrm{Co}}=111.5$ days and $M_{56}$ is the mass of ${ }^{56} \mathrm{Ni}$ that is exposed by the diffusion wave.

The depth of the diffusion wave tells us which part of the exploding star is being probed by the observations, and it is related to the time after explosion by (see Appendix C)

$$
\Delta M_{\text {diff }} \approx 8 \times 10^{-2} \frac{E_{51}^{0.44}}{\kappa_{0.1}^{0.88} M_{1}^{0.32}}\left(\frac{t}{1 \text { day }}\right)^{1.76} M_{\odot},
$$

where $\kappa_{0.1}=\kappa / 0.1 \mathrm{~cm}^{2} \mathrm{~g}^{-1}$ is the canonical value we use for the radioactively powered phase. This value for the opacity is motivated by the more detailed analysis of Pinto \& Eastman (2000). We discuss how this diffusion depth is adjusted by differences in the opacity, especially when the shallow layers become partially ionized, in more detail in Appendix C. For the estimates in this paper, we use quantities that roughly correspond to the $\mathrm{SNe} \mathrm{Ib} / \mathrm{c}$, but the same general arguments apply to $\mathrm{SNe} \mathrm{Ia}$ (as well as the rare SNe II that are radioactively powered) with different prefactors. To see how much these can vary for other progenitors, see Appendix C. Also note that Equation (8) becomes less accurate closer to the peak of the light curve, when $\Delta M_{\text {diff }}$ grows more slowly with time.

When powered by radioactive heating, the observed luminosity is no longer sensitive to the progenitor radius as can be seen by the lack of an explicit dependence on $R_{*}$ in Equation (8). Instead, it provides a direct measurement of the amount of ${ }^{56} \mathrm{Ni}$ (via Equation (6)) at the location of the diffusion wave. If the explosion time is known, Equation (8) provides $\Delta M_{\text {diff }}$ into which this ${ }^{56} \mathrm{Ni}$ is mixed. From this, the mass fraction $X_{56} \approx M_{56} / \Delta M_{\text {diff }}$ can be inferred as a function of the depth $\Delta M_{\text {diff }}$. Thus, in principle, detection of the rise of the ${ }^{56} \mathrm{Ni}$ light curve should provide an estimate of the distribution of ${ }^{56} \mathrm{Ni}$ (as attempted for the SN Ia 2011fe by Piro 2012), which is helpful for understanding the nature of the explosive burning and the outer structure of the progenitor.

\section{4. ${ }^{56}$ Ni Deeper than the Diffusion Depth: "Diffusive Tail"}

In practice, the exercise described above is not as straightforward as one might think. The reason is that if there is a steep increase in the ${ }^{56} \mathrm{Ni}$ abundance, then the assumption that the observed emission is generated only by the composition at the location of the diffusion wave may not be valid. Instead a "diffusive tail" of the energy released in ${ }^{56} \mathrm{Ni}$-rich layers deeper than the diffusion depth (shown as purple curves in Figure 1) may actually dominate over the energy released in ${ }^{56} \mathrm{Ni}$-poor layers shallower than the diffusion depth (shown as red curves in Figure 1). Next we estimate the contribution of this diffusive tail. To do this, we neglect the effect of expansion and adiabatic losses of the trapped radiation. This is appropriate since at any time the observed luminosity is dominated by energy that was deposited over the last dynamical timescale, during which expansion and adiabatic losses introduce only small corrections.

Consider first a deposit of ${ }^{56} \mathrm{Ni}$ at some depth $d$ in the star at $t=0$. The ${ }^{56} \mathrm{Ni}$ decays to produce gamma rays, which are absorbed and create thermal photons. The photons then spread due to diffusion, creating roughly a Gaussian distribution around the ${ }^{56} \mathrm{Ni}$. At each time $t$, the Gaussian has a width $\sqrt{K t}$, where $K$ is the diffusion coefficient, which is related to the diffusion time by $t_{\text {diff }}=d^{2} / K$. For such a distribution, there is a small, but non-zero, fraction of photons that escape from the star because they have diffused by a distance $>d$ from the ${ }^{56} \mathrm{Ni}$ depth. The fraction of photons released at $t=0$ that have reached the surface by time $t$ is roughly the integral of a Gaussian from $d$ to infinity, which results in

$$
\text { Escaping fraction } \approx \operatorname{erfc} \sqrt{t_{\text {diff }} / 2 t},
$$

where erfc is the complementary error function.

Photons are continuously emitted from the depth $d$, and thus the total number of photons that diffuse a distance $>d$ is the integral of erfc $\sqrt{t_{\text {diff }} / 2\left(t-t^{\prime \prime}\right)}$ with time from $t^{\prime \prime}=0$ to $t^{\prime \prime}=t$. The luminosity scales as the derivative of this integral total number of photons. Taking the derivative of an integral, we find that the luminosity scales proportional erfc $\sqrt{t_{\text {diff }} / 2 t}$. Putting these arguments together, if at some time $t^{\prime}$ the diffusion wave reaches a layer of ${ }^{56} \mathrm{Ni}$ that would be producing a luminosity $L_{56}\left(t^{\prime}\right)$, this implies for previous times $t<t^{\prime}$ that the diffusive tail from this layer also produces a luminosity,

$$
L_{\text {tail }}\left(t<t^{\prime}\right) \approx L_{56}\left(t^{\prime}\right) \frac{\epsilon(t)}{\epsilon\left(t^{\prime}\right)} \frac{\operatorname{erfc}\left(t^{\prime} / \sqrt{2} t\right)}{\operatorname{erfc}(1 / \sqrt{2})},
$$

where we have used $t_{\text {diff }} \propto \rho r^{2} \propto 1 / t$, so that $t_{\text {diff }}=t^{\prime 2} / t$. This is the appropriate normalization because, as we have described, the diffusion wave reaches the layer at time $t^{\prime}$ and therefore $t_{\text {diff }}=t^{\prime}$ when $t=t^{\prime}$.

From this discussion, one can see what we meant at the beginning of this section when we mentioned "a steep increase in the abundance of ${ }^{56} \mathrm{Ni}$." Consider the two ${ }^{56} \mathrm{Ni}$ distributions shown in Figure 2. In the top panel, the ${ }^{56} \mathrm{Ni}$ has a rather shallow distribution which produces the $L_{56}(t)$ (shown by the red curve). Therefore, if we consider the luminosity $L_{56}$ at some time $t^{\prime}$, and then trace back the diffusive tail implied from that depth using Equation (10) (shown as a purple curve), the diffusive tail always falls below the $L_{56}$ light curve. This is a case where the shallow ${ }^{56} \mathrm{Ni}$ prevents the diffusive tail from having a noticeable impact, and $M_{56}$ can be approximated from the observations. Furthermore, $X_{56} \approx M_{56} / \Delta M_{\mathrm{diff}}$ can be inferred as a function of time if the explosion time is well constrained. In the bottom panel of Figure 2, the ${ }^{56} \mathrm{Ni}$ has a steeper distribution. Now, when the diffusive tail is drawn back from a point at time $t^{\prime}$, it exceeds the $L_{56}$ light curve. In this latter case, the diffusive tail will dominate the observed rise, and this must be accounted for before attempting to infer $M_{56}$.

These conclusions are only approximate since we are focusing on the diffusive tail from a single depth. This is likely not too 


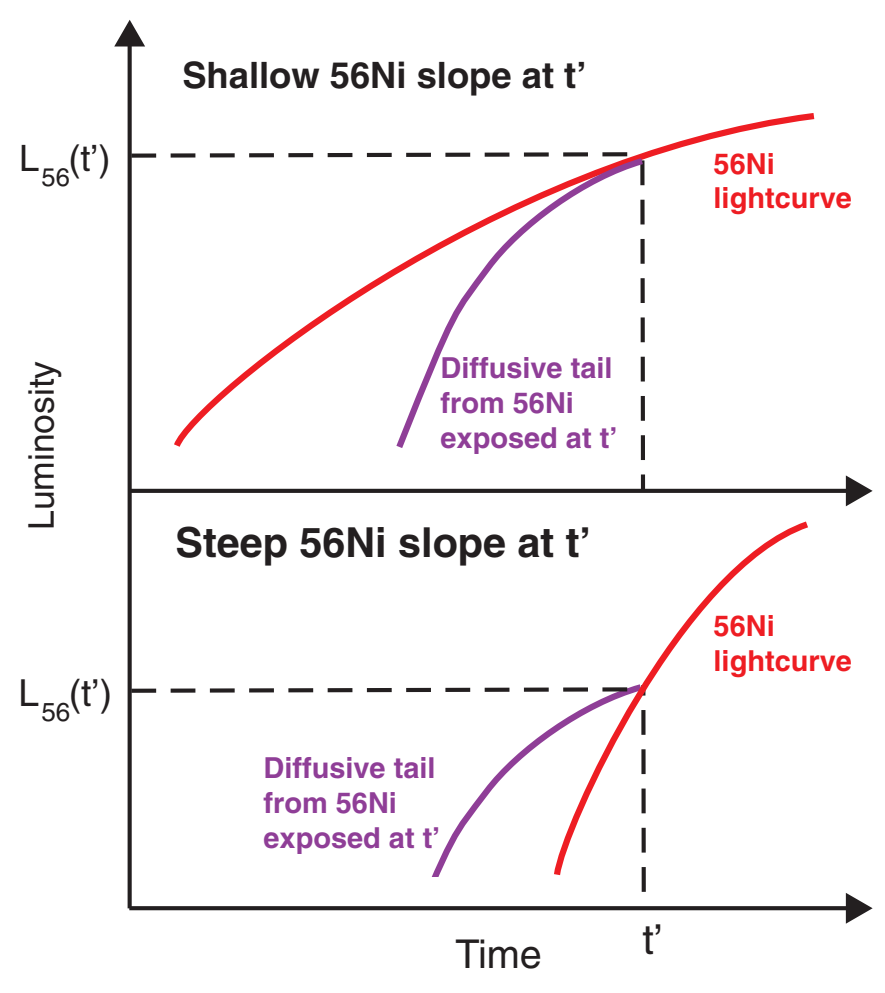

Figure 2. Schematic diagram showing how the slope of the ${ }^{56} \mathrm{Ni}$ distribution determines the relative importance of the diffusive tail. In both the top and bottom panels, a luminosity $L_{56}\left(t^{\prime}\right)$ would be produced at time $t^{\prime}$ from the diffusion wave probing the ${ }^{56} \mathrm{Ni}$ distribution. In the top panel, the slope of the ${ }^{56} \mathrm{Ni}$ is shallow, which produces a shallow slope for $L_{56}$ (shown by the red curve). If a diffusive tail luminosity is drawn back from this point (shown by the purple curve), it always falls below the ${ }^{56} \mathrm{Ni}$ light curve. Thus, the diffusive tail from this depth is not important. In the bottom panel, the slope of the ${ }^{56} \mathrm{Ni}$ distribution is steeper. Therefore, the diffusive tail from larger depths overpowers the heating from ${ }^{56} \mathrm{Ni}$ at shallower depths, and the diffusive tail from this depth impacts the light curve.

(A color version of this figure is available in the online journal.)

bad of a simplification, since the diffusive tails from ${ }^{56} \mathrm{Ni}$ at even larger depths are exponentially suppressed (by the scaling of the complementary error function). In detail, though, the luminosity of the diffusive tail should depend on the sum of contributions from all depths. We calculate and discuss the impact of this in Appendix B.

\subsection{The Importance of the Time of Explosion}

Our discussion thus far makes it clear that it is very important to know the time of explosion. It sets the time at which the thermal diffusion wave begins backing its way into the expanding ejecta, and from this at all later times it is roughly known what depths of the exploding star are being probed by the observations via Equation (8).

When the time of explosion is not known from direct detection of shock breakout or shock heating of the surface layers, our discussion of the early light curve should also provide some reason for caution. To illustrate the problem, in the top and middle panels of Figure 1 we compare the light curves for different ${ }^{56} \mathrm{Ni}$ depositions. In the top panel, the ${ }^{56} \mathrm{Ni}$ is deposited into rather shallow layers, therefore the timescale between the beginning of the explosion and the rise of the light curve is fairly short. In this case, the time of explosion could be reasonably well approximated by extrapolating the ${ }^{56} \mathrm{Ni}$ light curve back in time. In the middle panel, the ${ }^{56} \mathrm{Ni}$ is deposited in deeper layers, and correspondingly the delay between the shock heating and rising light curve is longer. In this case, extrapolating the ${ }^{56} \mathrm{Ni}$ light curve back in time would provide a poor estimate for the time of explosion. We are led to the following important conclusion: one cannot simply estimate the time of explosion by extrapolating the rising light curve back in time because its position relative to the moment of explosion depends on the depth of radioactive heating. This means that for events like SN $2011 \mathrm{fe}$, the constraints on $R_{*}$ cannot be as tight as previously reported (Bloom et al. 2012) when the explosion time is not known.

The earliest detection of the rising light curve from ${ }^{56} \mathrm{Ni}$ does not probe the shallowest layers of the star, but merely the radioactive heating of the diffusive tail from the shallowest deposits of ${ }^{56} \mathrm{Ni}$. For example, if the diffusion wave only reaches the heating after traveling $\sim 0.1 M_{\odot}$ below the exploding star's surface, then it will take $\sim 2$ days to detect this depth (using Equation (8)). Similar delays between the moment of explosion and the rising light curve are seen in the numerical work of Dessart et al. (2011).

\subsection{Clues about the Depth of ${ }^{56} \mathrm{Ni}$}

When the shock breakout and shock heating are not detected (as is often the case), additional information from color or, even better, spectroscopic observations can be used to break the degeneracy between the depth of ${ }^{56} \mathrm{Ni}$ and the time of explosion. We summarize some of the properties of SNe that are most useful for doing this in this section and the next.

In the bottom panel of Figure 1, we schematically show the time-dependent radius of the photosphere (orange curve) during an SN. For a polytropic index of $n=3$, the photospheric radius is (Rabinak \& Waxman 2011)

$$
r_{\mathrm{ph}}(t) \approx 3 \times 10^{14} \frac{\kappa_{0.1}^{0.11} E_{51}^{0.39}}{M_{1}^{0.28}}\left(\frac{t}{1 \text { day }}\right)^{0.78} \mathrm{~cm},
$$

where we have suppressed the dependence on the density structure factor $f_{\rho}$ and set it to 0.01 (see Appendix D). Even though $r_{\mathrm{ph}}$ is moving into the star as the ejecta expands, it is always moving out in an Eulerian frame. The observed color temperature is

$$
T_{c} \approx\left(\frac{L \tau_{c}}{4 \pi r_{\mathrm{c}}^{2} \sigma_{\mathrm{SB}}}\right)^{1 / 4} \gtrsim\left(\frac{L}{4 \pi r_{\mathrm{ph}}^{2} \sigma_{\mathrm{SB}}}\right)^{1 / 4},
$$

where $r_{c}$ is the color radius and $\tau_{c}$ is the optical depth at the color (thermalization) depth. The inequality comes from the fact that $\tau_{c} \geqslant 1$ and $r_{c} \leqslant r_{\mathrm{ph}}$. For typical parameters $\tau_{c}$ is not much larger than unity (e.g., Nakar \& Sari 2010) and so is $r_{\mathrm{ph}} / r_{c}$, so this inequality is also a fair approximation of $T_{c}$. Although $r_{\mathrm{ph}}$ roughly always scales as a power law with time (Equation (11)), $L(t)$ can vary greatly depending on the ${ }^{56} \mathrm{Ni}$ deposition. Thus, the color evolution during the rising phase provides the first clue on the ${ }^{56} \mathrm{Ni}$ depth. For deep ${ }^{56} \mathrm{Ni}$, the initial rising phase of $L$ is exponential, so from Equation (12) we see that $T_{c}$ should be rising as well. On the other hand, if ${ }^{56} \mathrm{Ni}$ is shallow then $L$ rises more gradually and $T_{c}$ is roughly constant or even slowly decreasing during the lightcurve rise.

In the bottom panel of Figure 1, we also show the photospheric velocity (green curve). Taking $v_{\mathrm{ph}}=r_{\mathrm{ph}} / t$, we find

$$
v_{\mathrm{ph}}(t) \approx 35,000 \frac{\kappa_{0.1}^{0.11} E_{51}^{0.39}}{M_{1}^{0.28}}\left(\frac{t}{1 \text { day }}\right)^{-0.22} \mathrm{~km} \mathrm{~s}^{-1} .
$$


Unlike the photospheric radius, the photospheric velocity is decreasing with time. This is because the photosphere is moving into the star in a Lagrangian sense, and reaching material that is moving at successively lower velocities. Furthermore, the time derivative of the photospheric velocity is $d v_{\mathrm{ph}} / d t \propto t^{-1.22}$. Therefore, we expect the change in the photospheric velocity to become more gradual with time.

Using the above arguments, we conclude that there are four main ways in which the depth of ${ }^{56} \mathrm{Ni}$ heating may be qualitatively inferred. All other things being equal, a larger ${ }^{56} \mathrm{Ni}$ depth implies the following.

1. The photospheric radius is larger when the ${ }^{56} \mathrm{Ni}$ heating gets out, and therefore the temperature will be lower.

2. The luminosity at early times is increasing faster than the radius expands, causing temperature to evolve bluer.

3. The photospheric velocities are lower.

4. The photospheric velocities evolve more slowly with time.

\subsection{The Minimum Explosion Time}

In additional to the qualitative correlations listed above, a single measurement of the photosphere velocity and color temperature during the rise can provide a strict, model-independent, upper limit to the time of explosion before that measurement. This is seen by first rewriting Equation (12) as

$$
L \approx 4 \pi r_{c}^{2} \sigma_{\mathrm{SB}} T_{c}^{4} / \tau_{c}
$$

If we define $v_{c}$ as the velocity of material at the color depth, then $r_{c} \approx v_{c} t_{\exp }$ where $t_{\exp }$ is the time since the explosion began. Putting these together, we estimate

$$
t_{\exp } \approx\left(\frac{L \tau_{c}}{4 \pi v_{c}^{2} \sigma_{\mathrm{SB}} T_{c}^{4}}\right)^{1 / 2}
$$

In general, $T_{c}$ can be measured directly from the observations, but any velocity that can be measured will be from an absorption feature shallower than the photosphere and generally $v_{\mathrm{ph}} \gtrsim v_{c}$. Furthermore, $\tau_{c}$ will be greater than unity but also difficult to infer just from the observation. It is therefore useful to have a quantity that can simply be estimated directly in terms of observable quantities,

$$
t_{\min } \equiv\left(\frac{L}{4 \pi v_{\mathrm{ph}}^{2} \sigma_{\mathrm{SB}} T_{c}^{4}}\right)^{1 / 2}=\frac{t_{\mathrm{exp}}}{\tau_{c}^{1 / 2}}\left(\frac{v_{c}}{v_{\mathrm{ph}}}\right) \lesssim t_{\mathrm{exp}} .
$$

Substituting typical values we find

$$
\begin{array}{r}
t_{\min }=4.3\left(\frac{L}{10^{42} \mathrm{erg} \mathrm{s}^{-1}}\right)^{1 / 2}\left(\frac{T_{c}}{10^{4} \mathrm{~K}}\right)^{-2} \\
\times\left(\frac{v_{\mathrm{ph}}}{10^{4} \mathrm{~km} \mathrm{~s}^{-1}}\right)^{-1} \text { days. }
\end{array}
$$

Therefore, $t_{\min }$ is an observable quantity that provides a modelindependent lower limit to the time of explosion. Another reason that $t_{\min }$ is especially useful is that it only requires that the velocity and temperature of the $\mathrm{SN}$ be obtained at a single time. Thus, when resources are limited, using Equation (17) is a helpful technique for learning a lot about the SN with minimal additional investment.

This concludes our discussion of the qualitative features of rising SN I light curves. In general, specific events may have details that we have not addressed, like non-spherical explosions

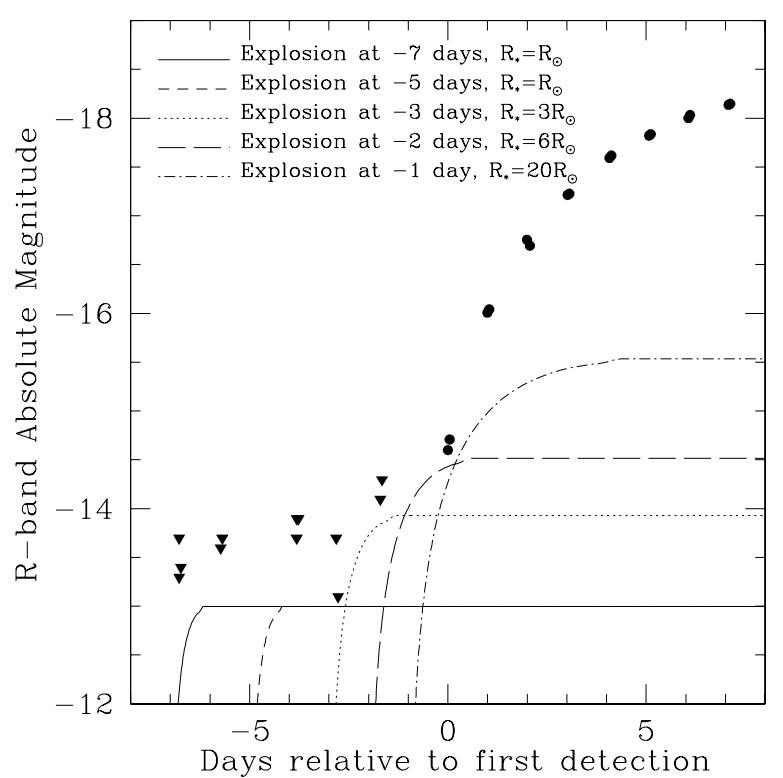

Figure 3. Circles and triangles plot the data for PTF 10vgv from Corsi et al (2012) for the detections and upper limits, respectively. The curves show theoretical calculations of the shock heating light curves using Equations (1) and (2), which are set to plateau at a temperature of $\approx 0.6 \mathrm{eV}$, for a range of explosion times and radii as labeled. In all cases, we fix $E=10^{51} \mathrm{erg}$ and $M=2 M_{\odot}$ for the sake of comparison. The main conclusion is that without direct identification of the time of explosion, the radius upper limit constraint can vary by an order of magnitude or more.

or complicated velocity profiles. But by clearly spelling out the chain of reasoning behind our conclusions, we provide rules of thumb that can be used to build intuition, even in cases that are somewhat more complicated. To illustrate how our arguments can be used in practice, in the next section we apply them to a recently discovered, well-studied SNe Ic.

\section{PTF 10vgv}

PTF 10vgv is an SN Ic that was discovered on 2010 September 14.1446 (UTC time) with the Palomar Oschin Schmidt 48 inch telescope (P48) by the PTF survey (Corsi et al. 2012). In a previous image taken on 2010 September 12.4830, it was not seen down to a limiting magnitude of $R>20.2$. Following detection, the $R$-band luminosity rises quickly to a peak $\approx 10$ days later. A single spectrum was taken $\approx 2$ days after detection. In light of what we have been discussing, it would have been ideal if the emission from shock heating could have been identified. It would have provided a measurement of the progenitor radius and the time of explosion, which could have been used to directly probe the ${ }^{56} \mathrm{Ni}$ deposition during the rising light curve. Although it is unfortunate that this was not available, this event is ideal as a test case for exploring what can be learned when the time of explosion is not known.

\subsection{Radius Constraints}

Even though shock-heated cooling was not observed, PTF $10 \mathrm{vgv}$ did have an early detection of the rising light curve and upper limits in the time before this. Using this information, interesting constraints on the progenitor's radius are still possible (Corsi et al. 2012). In Figure 3, we plot the observed $R$-band absolute magnitude of PTF 10vgv (circles), along with upper limits (triangle). These all assume a distance modulus of 34.05 and galactic extinction of $A_{R}=0.445 \mathrm{mag}$ (Schlegel et al. 1998). We then calculate the shock-heated cooling according to 


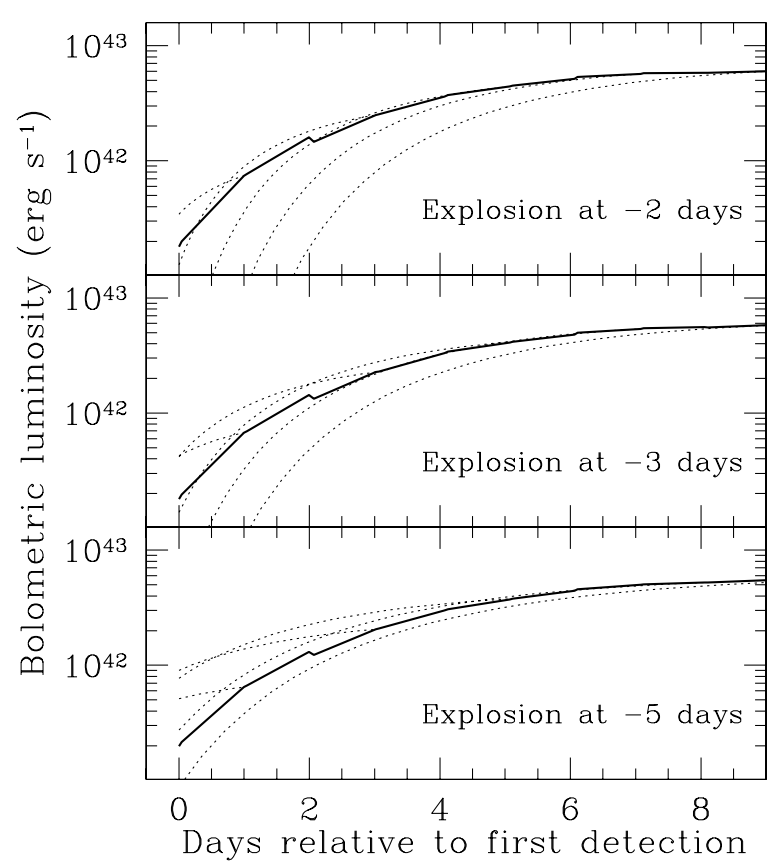

Figure 4. Bolometric luminosity of PTF 10vgv (thick, solid curves). From the top panel to the bottom panel, we consider explosion times of two, three, and five days before the first detection. In all cases, we fix $E=10^{51} \mathrm{erg}$ and $M=2 M_{\odot}$. For each light curve, at various times we consider what the diffusive tail coming from that depth would look like at earlier times (dotted lines) using Equation (10). From this comparison, we conclude that if the SN occurred recently (top panel), then only the earliest heating may be explained by a diffusive tail and the majority of the rising light curve directly probes the ${ }^{56} \mathrm{Ni}$ distribution. On the other hand, if the explosion occurred further in the past (the middle and bottom panels), then the majority of the rising light curve can be explained as a diffusive tail due to deep deposits of ${ }^{56} \mathrm{Ni}$. This example demonstrates the degeneracy between the time of explosion and the depth of ${ }^{56} \mathrm{Ni}$ if only a photometric light curve is available.

Equation (1), which transitions to the plateau phase given by Equation (5). We infer the corresponding $R$-band absolute magnitude using Equation (2), or a plateau temperature of $\approx 0.6 \mathrm{eV}$, along with $\mathrm{P} 48$-calibrated bolometric corrections provided by E. O. Ofek (2012, private communication). The resulting light curves are plotted alongside the data from PTF 10vgv in Figure 3 for a range of explosion times and progenitor radii. The conclusion is that without knowing the time of explosion, the radius can only be constrained to be $R_{*} \lesssim 1-20 R_{\odot}$. In general, the earlier the explosion time is the more stringent the constraints on the radius.

\subsection{Bolometric Light Curve}

Next we consider what can be learned about the mass and distribution of ${ }^{56} \mathrm{Ni}$ from the observed light curve. Before this can be done, there are two considerations that must be made. First, we need to convert the observed $R$-band magnitudes to a bolometric luminosity. We solve for this using

$$
L \approx 4 \pi r_{c}^{2} \sigma_{\mathrm{SB}} T_{c}^{4} / \tau_{c} \approx 10^{\left(M_{R, \odot}-M_{R}-\mathrm{BC}\right) / 2.5} L_{\odot} .
$$

Since BC depends on $T_{c}$, we can self-consistently find $T_{c}$ and thus $L$ at any given time. For our estimates here we simply take $\tau_{c} \sim 1$ and $r_{c} \sim r_{\mathrm{ph}}$ as given by Equation (11) to produce the thick, solid curves in Figure 4. Although not physically accurate, these estimates are adequate since the bolometric luminosity is found to be fairly robust. Once again, we must take into account that the time of explosion is not known, so we consider three example explosion times.

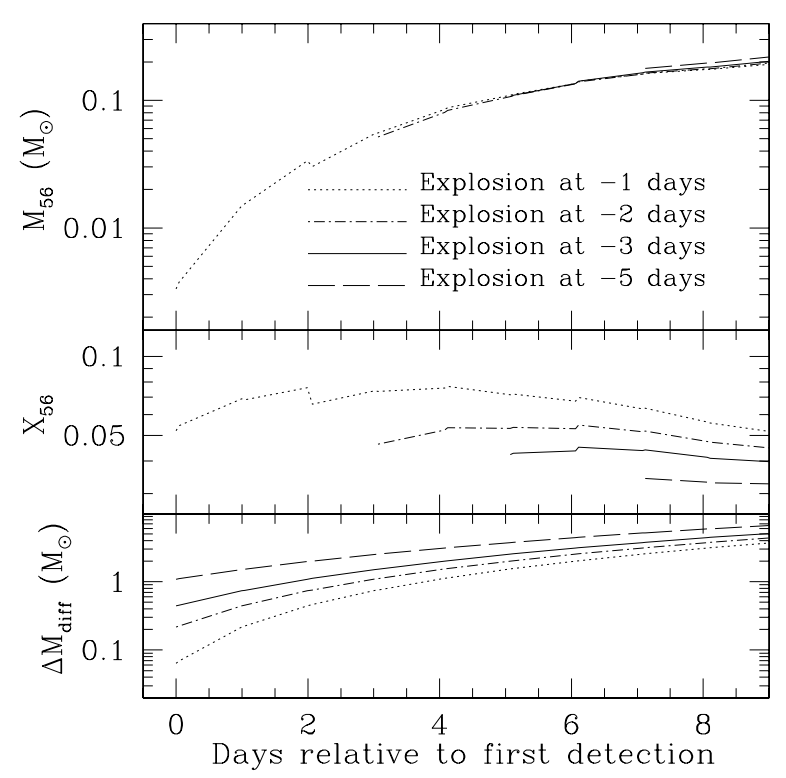

Figure 5. Inferred mass, mass fraction, and depth of ${ }^{56} \mathrm{Ni}$ in PTF $10 \mathrm{vgv}$ for different explosion times. The ${ }^{56} \mathrm{Ni}$ mass $M_{56}$ is inferred from Equation (6), and we only plot $M_{56}$ and $X_{56}$ for times at which the bolometric luminosity cannot be explained as a diffusive tail according to Figure 4. The mass fraction is estimated as $X_{56} \approx M_{56} / \Delta M_{\text {diff }}$, where the diffusion depth is calculated according to Equation (8).

The second consideration we must make before attempting to associate the bolometric light curve with the ${ }^{56} \mathrm{Ni}$ distribution is the impact of a diffusive tail. Assuming that the bolometric light curves in Figure 4 are representative of the ${ }^{56} \mathrm{Ni}$ distribution, we can then ask, for a given luminosity at a given time, what is the implied diffusive tail at earlier times? The dotted lines in Figure 4 show the diffusive tails originating from various times using Equation (10). If the dotted line sits below the solid curve, this means that the diffusive tail is insufficient to explain the bolometric light curve at this depth, and thus the bolometric light curve represents direct heating from ${ }^{56} \mathrm{Ni}$ (as shown in the top diagram in Figure 2). On the other hand, if the dotted line sits above the solid curve, then the diffusive tail from that point overestimates the earlier bolometric light curve and luminosity at that time cannot be from direct heating of ${ }^{56} \mathrm{Ni}$ at the diffusion depth (as shown in the bottom diagram in Figure 2).

The conclusion from this comparison is that it is difficult to determine which of these three explosion times are more accurate from just this information. If the $\mathrm{SN}$ occurred recently (top panel of Figure 4), then only the earliest heating is explained by a diffusive tail and we would infer that the majority of the rising light curve directly probes the ${ }^{56} \mathrm{Ni}$ distribution. On the other hand, if the explosion occurred further in the past (the middle and bottom panels of Figure 4), then the majority of the rising light curve is explained as a diffusive tail due to deep deposits of ${ }^{56} \mathrm{Ni}$. This is consistent with our discussion in Section 2.6 that there is a degeneracy between these two limits unless more information is available.

Nevertheless, we can still try to constrain the mass and distribution of ${ }^{56} \mathrm{Ni}$ as a function of the explosion time, the results of which are shown in Figure 5. In the top panel, we plot the mass of ${ }^{56} \mathrm{Ni}$ inferred from Equation (6). This is only plotted for depths at which the bolometric luminosity cannot be explained by a diffusive tail using Figure 4. In the middle panel, we plot the mass fraction $X_{56}$, and in bottom panel the thermal diffusion depth using Equation (8). 


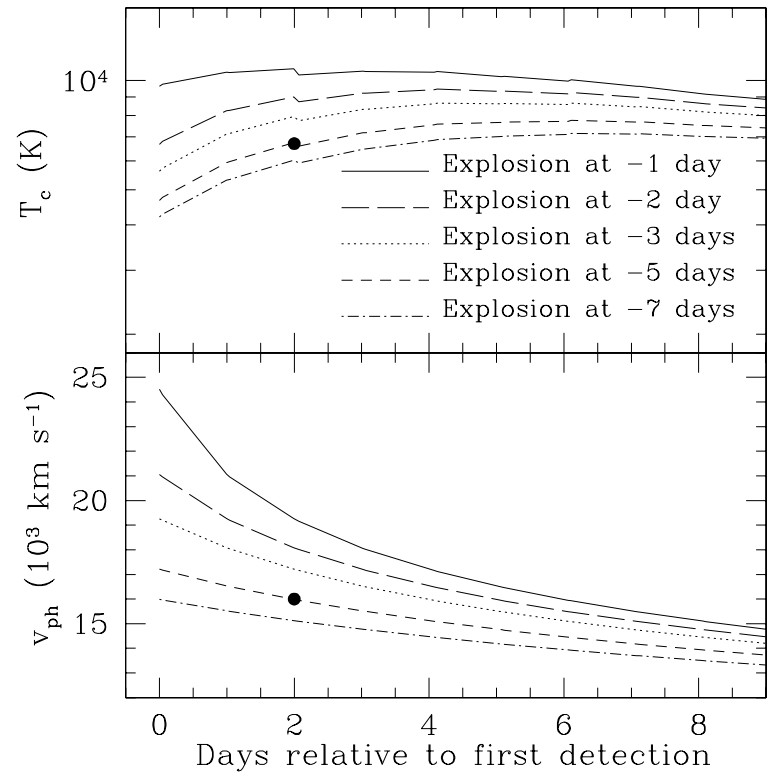

Figure 6. Temperature and velocity evolution for PTF $10 \mathrm{vgv}$ when fitting a temperature of $6700 \mathrm{~K}$ and velocity of $16,000 \mathrm{~km} \mathrm{~s}^{-1}$ at two days past first detection (shown as filled circles). In this particular case, the best fits occur for $E_{51}=0.41 M_{1}^{0.72}$. The curves are theoretical calculations assuming different explosion times as labeled, which demonstrates that an explosion time of $\sim 5$ days before first detection is favored.

\subsection{Temperature and Velocity Constraints}

We next consider what additional information can be learned about PTF 10vgv from temperature and velocity measurements. Unfortunately, the available data during the rise are rather limited. Corsi et al. (2012) mention a $16,000 \mathrm{~km} \mathrm{~s}^{-1}$ $\mathrm{Si}$ II absorption feature (which is typically associated with the photosphere; Tanaka et al. 2008) observed on 2010 September 16 , which corresponds to $\approx 2$ days after discovery. From the spectrum taken on that same day, by eye one can see that it roughly peaks at $\sim 4300 \AA$, which, assuming a blackbody spectrum, corresponds to a temperature of $\sim 6700 \mathrm{~K}$. But we must be cautious. In the study of SN 1994I by Sauer et al. (2006), a spectrum with a similar peak is observed at eight days post-explosion, and their detailed models infer a temperature of $\sim 10,000 \mathrm{~K}$. As we describe next, if the correct temperature were known, then tight constraints could be placed on the time of explosion and thus the other properties of PTF 10vgv. But, given that the temperature cannot be extracted from the data without a detailed spectral modeling, which is beyond the scope of this paper, we separately consider both the cases of $6700 \mathrm{~K}$ and $10,000 \mathrm{~K}$ and discuss the implications.

Using Equation (17), we estimate the minimum time of the explosion. For $T_{c}=6700 \mathrm{~K}, v_{\mathrm{ph}}=16,000 \mathrm{~km} \mathrm{~s}^{-1}$, and $L \approx 1.5 \times 10^{42} \mathrm{erg} \mathrm{s}^{-1}$ (taken from Figure 4), we find $t_{\min } \sim 7$ days. Therefore, if the cooler temperature is the correct one then the explosion must have occurred $\sim 5$ days or more before the first detection. On the other hand, for $T_{c}=10,000 \mathrm{~K}$ we estimate $t_{\min } \sim 3$ days and the explosion must have occurred $\sim 1$ day or more before the first detection.

To test these conclusions, we perform a detailed fit of the temperature, velocity, and bolometric luminosity constraints simultaneously. This is done by varying $E, M$, and the time of explosion over a wide range of values and identifying which best fit the constraints. The result for either $6700 \mathrm{~K}$ or $10,000 \mathrm{~K}$ is that a fit can only be obtained when $E \propto M^{0.72}$ because this

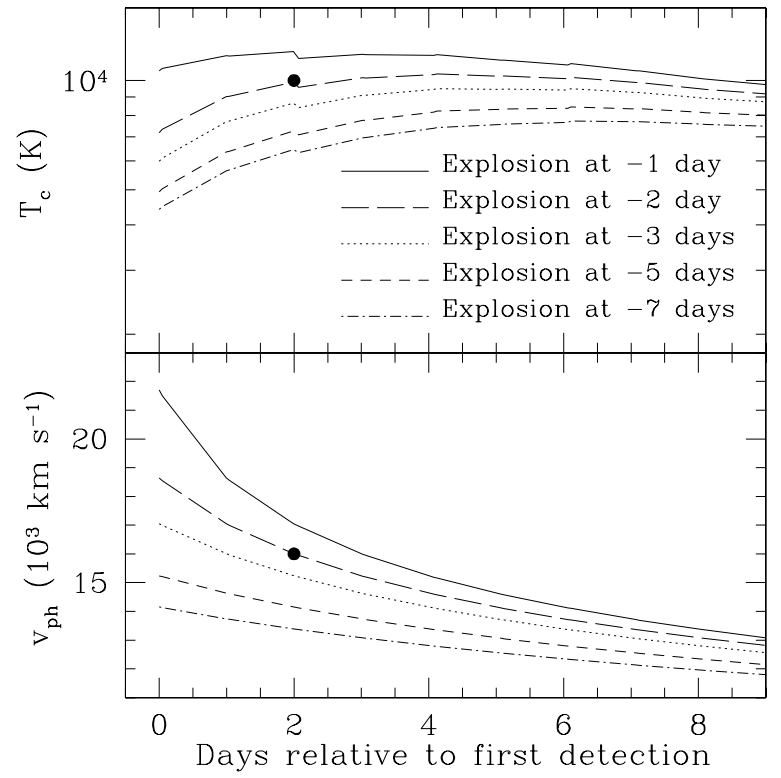

Figure 7. Same as Figure 6, but this time fitting a temperature of $10,000 \mathrm{~K}$. Now the best fits occur for $E_{51}=0.30 M_{1}^{0.72}$. In this hotter case, the explosion time is constrained to be $\sim 2$ days before first detection.

fixes the normalization of the velocity (see Equation (13)). But, for a given temperature, only a very narrow range of explosion times are consistent with the data. In Figures 6 and 7, we plot the results of our fitting. These show that for a temperature of $6700 \mathrm{~K}$ or $10,000 \mathrm{~K}$, the explosion much have occurred $\sim 5$ days or $\sim 2$ days prior to first detection, respectively. These match the values of $t_{\text {min }}$ estimated before.

Besides showing how the explosion time can be constrained, Figures 6 and 7 highlight many of the general trends that we discussed in Section 2 when considering how different explosion times impact various observables of the SN. For a more recent explosion time, the gradient in $v_{\mathrm{ph}}$ is greater and the temperature is higher and decreasing. In contrast, for an explosion time more distant in the past, $T_{c}$ is lower and increasing for a longer time. If merely a couple of data points were available, these trends could be identified in the observations, and even tighter constraints could be placed on PTF 10vgv. Nevertheless, it is powerful that even a single velocity and temperature measurement provides such stringent constraints. SNe Ia tend to have more early data available than $\mathrm{SNe} \mathrm{Ib} / \mathrm{c}$, and we consider some of these events in forthcoming work.

\subsection{Progenitors}

Our conclusions from the previous sections show just how greatly our inferences about the progenitor can vary depending on the explosion time. These are summarized as follows.

1. If the temperature at 2 days past first detection is $\sim 6700 \mathrm{~K}$, then the explosion occurred $\sim 5$ days or more before first detection. The radius is constrained to be $\lesssim 1 R_{\odot}$ and the ${ }^{56} \mathrm{Ni}$ must be located much deeper in the ejecta. Also the ejecta is likely of higher mass since it takes longer to reach the $\mathrm{SN}$ peak.

2. If the temperature at 2 days past first detection is $\sim 10,000 \mathrm{~K}$, then the explosion occurred merely $\sim 2$ days before first detection. The radius is constrained to be $\lesssim 6 R_{\odot}$ and the ${ }^{56} \mathrm{Ni}$ must be located fairly shallowly. Also the ejecta is likely of lower mass. 
Thus we are left with two seemingly opposite conclusions about almost all the aspects of the ejecta just from opposite assumptions about the time of explosion. We hope that a proper modeling of the available spectrum, which we do not carry out here, can provide the correct temperature and decide between these two options. We next discuss the implications for the progenitor star that produced PTF 10vgv in each case.

Since the progenitors of such SN Ic are hydrogen-stripped stars, the models of Yoon et al. (2010) are a helpful starting place. The most striking feature of these progenitors is the inverse relationship between mass and radius that is shown in their Figure 12. If PTF 10vgv had a radius $\lesssim 1 R_{\odot}$, it is strongly inconsistent with any of the binary models below $M_{f} \lesssim 7 M_{\odot}$, where $M_{f}$ is the final mass at the time of explosion, which all have radii larger than $\sim 2 R_{\odot}$. Their helium star models (i.e., Wolf-Rayet progenitors) have smaller radii and may be consistent with $M_{f} \gtrsim 6-8 M_{\odot}$, depending of the mass-loss prescription employed. ${ }^{6}$ Other progenitors that are consistent with such small radii are the gamma-ray burst models of Yoon et al. (2006) with $M_{f} \gtrsim 12 M_{\odot}$, but these probably are not applicable to PTF 10vgv.

Combining the generally large mass inferred for a smallradius progenitor with an ejecta mass of $\sim 3-5 M_{\odot}$ (estimated from $\Delta M_{\text {diff }}$ at peak), the remnant mass must be $\gtrsim 2-3 M_{\odot}$. This is near the range of the maximum mass of normal neutron stars (Lattimer \& Prakash 2001). Would this imply that this event led to the formation of a black hole? Given the number of estimates that have been made throughout our analysis, it is difficult to make this conclusion with such certainty. But this does demonstrate how our analysis can make interesting connections between observations on one hand and the detailed simulations of progenitors on the other.

For the case where $R_{*} \lesssim 6 R_{\odot}$, Yoon et al. (2010) show that such radii are naturally expected for progenitors in a mass range of $M_{f} \sim 4-5 M_{\odot}$, and it is easier to reconcile the ejecta mass estimate with formation of a neutron star remnant. A less massive progenitor with a large radius has been predicted to have mixing of ${ }^{56} \mathrm{Ni}$ into the outer layers by Rayleigh-Taylor instability during the SN explosion (Hachisu et al. 1991; Joggerst et al. 2009), similar to what we infer for a recent explosion (Figure 5). So even in this case there are interesting correlations and implications for the explosion mechanism that can be investigated.

Finally, it is worth discussing the inferences that can be made from the fact that PTF 10vgv was classified as an SN Ic. Dessart et al. (2012) studied the influence of ${ }^{56} \mathrm{Ni}$ mixing on the ejecta of $\mathrm{SNe} \mathrm{Ib} / \mathrm{c}$, and found that it strongly impacts the observed spectral features. Chief among these are the He I lines, which require non-thermal electrons that are excited by $\gamma$-ray lines from ${ }^{56} \mathrm{Ni}$ and ${ }^{56} \mathrm{Co}$ when the helium mass fraction is $\lesssim 0.5$ (Dessart et al. 2011). This means that the same ejecta with a significant amount of helium can produce either an SN Ib if the ${ }^{56} \mathrm{Ni}$ mixing is strong or an $\mathrm{SN}$ Ic if the ${ }^{56} \mathrm{Ni}$ mixing is weak. If PTF 10vgv were constrained to have a recent explosion with strong ${ }^{56} \mathrm{Ni}$ mixing, we would be forced to conclude that its outer layers are very helium-poor. In the future, it would be informative to repeat our analysis on an $\mathrm{SN}$ Ib to see if shallow ${ }^{56} \mathrm{Ni}$ can be inferred to make the He I visible.

\footnotetext{
6 In previous calculations of helium stars, such as by Woosley et al. (1995), smaller radii are generally seen. This difference is thought to be due to both different mass-loss rates and updated opacities; Yoon et al. (2010) use opacities from Iglesias \& Rogers (1996).
}

\section{CONCLUSIONS AND DISCUSSION}

We have discussed the photometric rising light curves of $\mathrm{SNe}$ I to investigate what can and cannot be learned from these observations. We provided a detailed summary of the various stages that determine the early light curve, and how their relative contributions are impacted by ${ }^{56} \mathrm{Ni}$ mixing and uncertainties in the explosion time. We then looked at a particular SN Ic event PTF 10vgv in some detail. Even with no direct constraint on the explosion time, and from just an $R$-band photometric light curve and a single estimate of the velocity and temperature of the ejecta, we argued that the explosion time could be constrained, which would have had a number of implications for the progenitor that we discussed.

Our investigation demonstrates the kinds of connections that can be made between observations, explosion calculations, and progenitor models. These strategies will be key for maximizing the science learned from wide field, high cadence optical surveys as they continue to find more $\mathrm{SNe}$ at earlier times. To facilitate such connections in the future, we summarize our conclusions below. We also highlight observational information that we deem especially important, and which should be considered of high priority when investigating a rising light curve on limited resources.

1. An SN may exhibit a dark phase between the moment of explosion and the rise of the ${ }^{56} \mathrm{Ni}$ light curve (likely first reflecting the impact of the diffusive tail). This means that extrapolating the ${ }^{56} \mathrm{Ni}$ light curve back in time is not a reliable method for estimating the time of explosion, and that without a known explosion time, constraints on $R_{*}$ are less stringent.

2. Even though shock breakout may only emit at short wavelengths and may be too short-lived for detection in most circumstances, the UV/optical detection of shock-heated cooling phase can be just as useful for putting constraints on the progenitor radius.

3. If caught when rising (Equation (3)), shock-heated cooling can also identify the time of explosion. Conversely, if the $\mathrm{UV} /$ optical rise is steeper than $\sim t^{1.5}$, then this argues that the shock-heated cooling is not being observed, and the explosion time is not well constrained. Due to a possible dark phase, data right before the ${ }^{56} \mathrm{Ni}$ light curve may not be sufficient to catch this rise that instead may occur $\sim 1-5$ days earlier.

4. If the time of explosion is well constrained, then the ${ }^{56} \mathrm{Ni}$ mixing can be extracted from the bolometric luminosity in the following way. First use Equation (6) to find $M_{56}(t)$ assuming there is no contribution from the diffusive tail. Then at any time $t^{\prime}$ find the diffusive tail contribution from $M_{56}(t)$ at $t<t^{\prime}$ (Equation (10)). If this contribution falls below the actual luminosity then $M_{56}(t)$ is a reliable estimate of the ${ }^{56} \mathrm{Ni}$ mass that mixed into $\Delta M_{\text {diff }}(t)$. Otherwise it is not. An upper limit on $M_{56}(t)$ can be derived then by finding the largest ${ }^{56} \mathrm{Ni}$ mass that produces a diffusive tail that falls below the observed luminosity.

5. If the time of explosion is unknown, having even a single temperature and velocity measurement during the rise can go a long way toward supplementing the photometric data and provide strong constraints on the time of explosion using Equation (17).

6 . Having $\gtrsim 2$ temperature and velocity measurements during the rise will allow the time evolution of each to be constrained in more detail. Knowing the time evolution 
of the temperature is especially useful because it provides a check of any conclusions about ${ }^{56} \mathrm{Ni}$ mixing.

7. A future goal of observations should be to obtain similar coverage of $\mathrm{SNe} \mathrm{Ib} / \mathrm{c}$ with strong ${ }^{56} \mathrm{Ni}$ mixing. Such events would likely be classified as $\mathrm{SNe} \mathrm{Ib}$ unless completely devoid of helium (Dessart et al. 2012) and should never show a shock-heated cooling phase. This would be an important test of whether all the telltale signs of ${ }^{56} \mathrm{Ni}$ mixing are present as we have outlined.

Although the scalings we have used are fairly robust, the numerical prefactors come from semi-analytic work that is only approximately correct. A useful future project would therefore be to calibrate our prefactors against more detailed numerical calculations, in particular the photospheric velocity, color temperature, and diffusion depth. As highlighted by Dessart et al. (2012), the opacities for these ejecta can become complicated, so the various fits may only be applicable over different subsets of parameter space in composition and temperature. Nevertheless, the resulting collection of relations would be useful tools for quickly estimating properties of the explosions and the progenitors from future observations.

We thank the anonymous referee for helpful comments and suggestions. We thank Eran Ofek for assistance in implementing the bolometric corrections for the P48, Avishay Gal-Yam for providing detailed comments, and Alessandra Corsi for helping with information about PTF 10vgv. We also thank Luc Dessart, Peter Goldreich, Christian Ott, and Re'em Sari for thoughtful discussions. A.L.P. was supported through NSF grants AST1212170, PHY-1151197, and PHY-1068881, NASA ATP grant NNX11AC37G, NSF grant AST-0855535, and by the Sherman Fairchild Foundation. E.N. was partially supported by an ERC starting grant (GRB-SN 279369).

\section{APPENDIX A}

\section{SHOCK-HEATED COOLING IN THE RAYLEIGH-JEANS TAIL}

At early times, when the light curve is dominated by shockheated cooling of ejecta and before ${ }^{56} \mathrm{Ni}$ heating has become important, the light curve can rise in the optical/UV even if the bolometric luminosity is declining. This is possible as long as the optical/UV emission is in the Rayleigh-Jeans tail. In this limit

$$
L_{\mathrm{opt} / \mathrm{UV}} \propto \frac{r_{c}^{2} T_{c}}{\tau_{c}},
$$

where $r_{c}$ is the color radius and $\tau_{c}$ is the optical depth at the color depth. Using the scalings from Equations (20) and (21) of Nakar \& Sari (2010), we find that $r_{c} \propto t^{0.83}$ and $\tau_{c} \propto t^{-0.44}$ for a polytropic index of $n=3$. Combining this with $T_{c}$ from Equation (2) above, this gives $L_{\mathrm{opt} / \mathrm{UV}} \propto t^{1.5}$ as summarized in Section 2.2. Therefore when a light curve rises faster than $t^{1.5}$ it is strong evidence that something other than shock heating is provided the observed energy (although this conclusion is not foolproof, because in principle velocity gradients may differ from $v_{s} \propto \rho^{-0.19}$ as we assume).

\section{APPENDIX B}

\section{THE TOTAL CONTRIBUTION FROM ${ }^{56} \mathrm{Ni}$ HEATING}

As discussed in Sections 2.3 and 2.4, the total observed luminosity from ${ }^{56} \mathrm{Ni}$ heating consists of both direct heating and diffusive tail components. For the majority of the analysis in this paper, we focus on the local contribution of these two components. This is adequate for the discussions we present, most of which is meant to be schematic. Here, we consider these two components in more detail in an attempt to better quantify the impact of these approximations.

In particular, these components are not from a single location, but are rather integrals over a range of depths. In the case of the direct heating component, the heating roughly comes from the surface all the way down to the diffusion depth, thus

$$
L_{56, \text { total }}(t)=\int_{0}^{t} X_{56}\left(t^{\prime}\right) \frac{\partial \Delta M_{\mathrm{diff}}}{\partial t^{\prime}} \epsilon(t) d t^{\prime}
$$

where $X_{56}(t)$ is the mass fraction of ${ }^{56} \mathrm{Ni}$ at the depth of the diffusion wave at time $t$. By setting the lower integration limit to zero, we are ignoring the fact that at sufficiently shallow depths the gamma rays from radioactive heating may not thermalize. The total diffusive tail component is the integral over all of the diffusive tails from heating deeper than $\Delta M_{\text {diff }}$. Such an integral can be approximated as

$$
\begin{aligned}
L_{\text {tail,total }}(t)= & \int_{t}^{t_{\text {peak }}} X_{56}\left(t^{\prime}\right) \frac{\partial \Delta M_{\text {diff }}}{\partial t^{\prime}} \epsilon(t) \\
& \times \frac{\operatorname{erfc}\left(t^{\prime} / \sqrt{2} t\right)}{\operatorname{erfc}(1 / \sqrt{2})} d t^{\prime}
\end{aligned}
$$

where we take the limit of the integration to be the time of peak $t_{\text {peak }}$, since this roughly corresponds to when the diffusion wave has made its way completely through the ejecta. Making use of the power-law time dependence of the diffusion depth $\left(\Delta M_{\text {diff }} \propto t^{1.76}\right)$ from Equation (8), Equations (B1) and (B2) can be rewritten as

$$
L_{56, \text { total }}(t)=1.76 L_{56}(t) \int_{0}^{t} \frac{X_{56}\left(t^{\prime}\right)}{X_{56}(t)}\left(\frac{t^{\prime}}{t}\right)^{1.76} \frac{d t^{\prime}}{t^{\prime}},
$$

and

$$
\begin{aligned}
L_{\text {tail,total }}(t) \approx & 1.76 L_{56}(t) \int_{t}^{t_{\text {peak }}} \frac{X_{56}\left(t^{\prime}\right)}{X_{56}(t)}\left(\frac{t^{\prime}}{t}\right)^{1.76} \\
& \times \frac{\operatorname{erfc}\left(t^{\prime} / \sqrt{2} t\right)}{\operatorname{erfc}(1 / \sqrt{2})} \frac{d t^{\prime}}{t^{\prime}}
\end{aligned}
$$

respectively, where $L_{56}(t) \equiv X_{56}(t) \Delta M_{\mathrm{diff}}(t) \epsilon(t)$ is the local heating rate from ${ }^{56} \mathrm{Ni}$ as was presented in Equation (6).

When actually performing calculations with this framework, it is useful to write these expressions in dimensionless forms. First, since the luminosity has no contribution from the diffusive tail once the diffusion wave has traveled through the ejecta, we define the luminosity at peak to be $L_{\text {peak }} \equiv L_{56 \text {,total }}\left(t=t_{\text {peak }}\right)$. Next, we let $x \equiv t / t_{\text {peak }}$ and $x^{\prime} \equiv t^{\prime} / t_{\text {peak }}$, where $x$ and $x^{\prime}$ vary from 0 to 1 . Finally, we define the ratio of the local heating rate to $L_{\text {peak }}$ as

$$
\begin{aligned}
\Lambda(x) & \equiv 1.76 \frac{L_{56}(x)}{L_{\text {peak }}} \\
& =\frac{\epsilon(x)}{\epsilon(1)}\left[\int_{0}^{1} \frac{X_{56}\left(x^{\prime}\right)}{X_{56}(x)}\left(\frac{x^{\prime}}{x}\right)^{1.76} \frac{d x^{\prime}}{x^{\prime}}\right]^{-1} .
\end{aligned}
$$



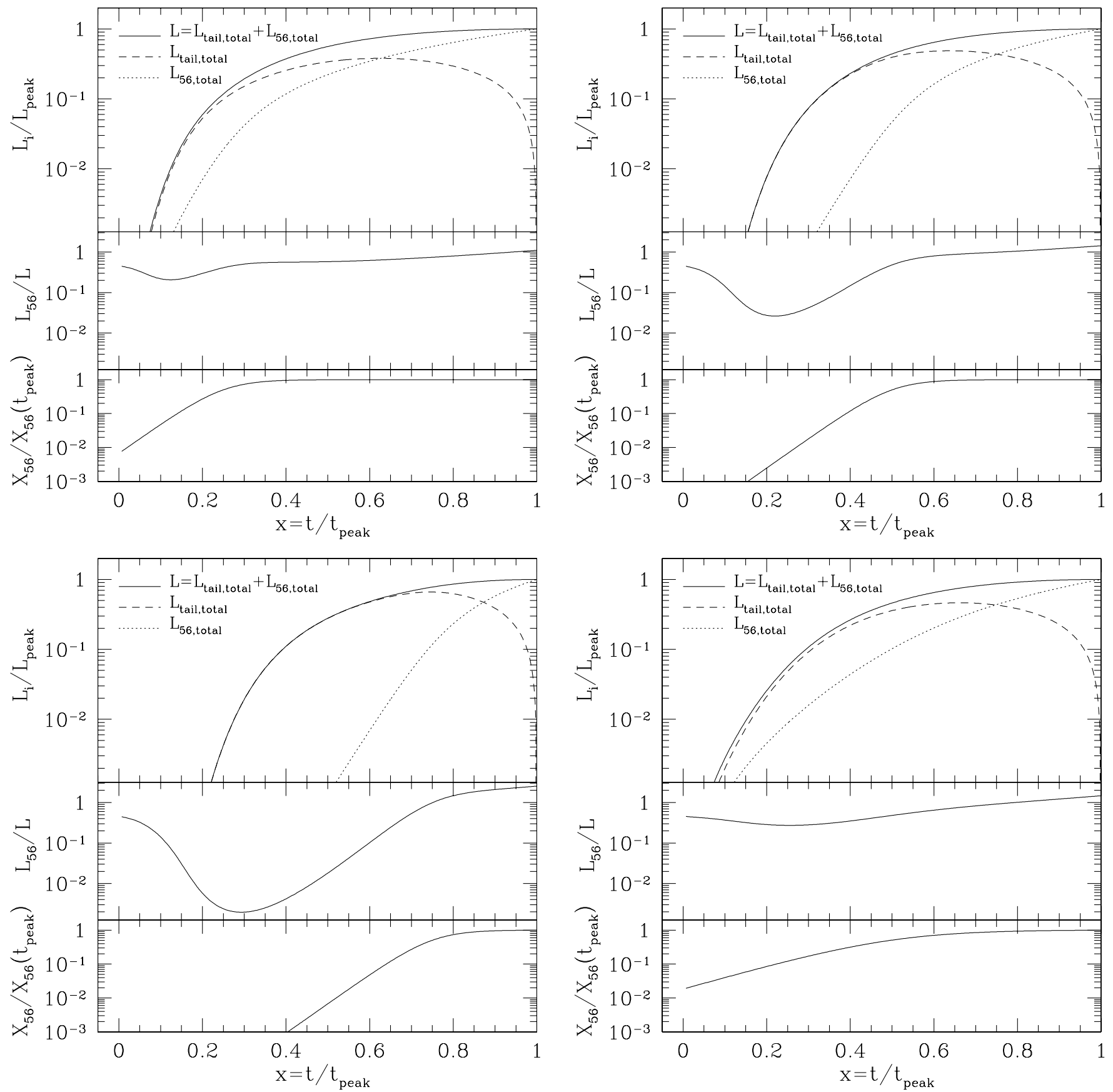

Figure 8. Comparisons on the light curves (shown in each top panel) for different distributions of ${ }^{56} \mathrm{Ni}$ (shown in the bottom panels), when the full integrals are used for both the direct and diffusive tail heating according to Equation (B6). In the middle panels, we plot the ratio of the local heating rate $L_{56}$, which is usually what we wish to infer from the observations, to the total luminosity $L$.

The ratio of the observed time-dependent luminosity to $L_{\text {peak }}$ is then

$$
\begin{aligned}
\frac{L(x)}{L_{\text {peak }}}= & \frac{L_{56, \text { total }}(x)+L_{\text {diff,total }}(x)}{L_{\text {peak }}} \\
= & \Lambda(x) \int_{0}^{x} \frac{X_{56}\left(x^{\prime}\right)}{X_{56}(x)}\left(\frac{x^{\prime}}{x}\right)^{1.76} \frac{d x^{\prime}}{x^{\prime}} \\
& +\Lambda(x) \int_{x}^{1} \frac{X_{56}\left(x^{\prime}\right)}{X_{56}(x)}\left(\frac{x^{\prime}}{x}\right)^{1.76} \frac{\operatorname{erfc}\left(x^{\prime} / \sqrt{2} x\right)}{\operatorname{erfc}(1 / \sqrt{2})} \frac{d x^{\prime}}{x^{\prime}} .
\end{aligned}
$$

In this form the right-hand side is dimensionless and only depends on the ${ }^{56} \mathrm{Ni}$ distribution.
In Figure 8, we plot the results of evaluating Equation (B6) for various distributions of $X_{56}$. In the first three plots, we keep the shape of the increase of $X_{56}$ the same, but vary its depth, increasing in depth from the top left plot to the top right plot to the bottom left plot. In the bottom right plot, we consider a flatter distribution of ${ }^{56} \mathrm{Ni}$. The top panel of each plot shows the various contributions to the light curve as labeled. The middle panels show the ratio of the local heating rate $L_{56}$ to the total luminosity. The bottom panel shows the $X_{56}$ distribution.

The middle panels are key to comparing all the plots in Figure 8 . The dimensionless ratio $L_{56} / L$ represents how well the observed luminosity compares to the local heating rate that we would like to infer so as to probe the ${ }^{56} \mathrm{Ni}$ distribution. When $L_{56} / L \sim 1$, then the observed luminosity should roughly 
tell us how much ${ }^{56} \mathrm{Ni}$ is present. On the other hand, when $L_{56} / L \ll 1$, then the true amount of ${ }^{56} \mathrm{Ni}$ is much less (by a factor of $L_{56} / L$ ) than what would be inferred from the observations. These examples confirm our general conclusion that the impact of the tail is diminished at times closer to the lightcurve peak. Furthermore, the more centrally concentrated the ${ }^{56} \mathrm{Ni}$ distribution, the larger the impact of the diffusive tail, as can be seen in the bottom left plot. In such cases, one must be careful when attempting to infer the ${ }^{56} \mathrm{Ni}$ distribution at shallow depths.

\section{APPENDIX C}

\section{THE DIFFUSION DEPTH}

Here, we derive the depth of the diffusion wave as a function of time, as was used in Equation (8). Our approach here is similar to that presented by Rabinak \& Waxman (2011), with a few changes of numerical factors.

The pre-explosion density profile of the star is approximated as

$$
\rho_{0}\left(r_{0}\right)=\rho_{1 / 2} \delta^{n},
$$

where $\rho_{1 / 2}$ is the density at half the radius, $\delta=1-r_{0} / R_{*}$ is the fractional radius, and $n$ is the polytropic index. The fraction of ejecta mass lying above a radius $r_{0}$ is

$$
\delta_{m}=\frac{1}{M} \int_{(1-\delta) R_{*}}^{R_{*}} 4 \pi r_{0}^{2} \rho_{0}\left(r_{0}\right) d r_{0} \approx \frac{3 f_{\rho}}{n+1} \delta^{n+1},
$$

so that the mass depth is $\Delta M=\delta_{m} M$.

The velocity profile from the passage of the SN shock is taken from Matzner \& McKee (1999), who use an interpolation between the Sedov-von Neuman-Taylor and the Gandel'Man-Frank-Kamenetskii-Sakurii self-similar solutions (Von Neumann 1947; Sedov 1959; Taylor 1950; Gandel'Man \& Frank-Kamenetskii 1956; Sakurai 1960). Near the surface of the star this gives

$$
v_{s}=A_{v}\left(\frac{E}{M}\right)^{1 / 2}\left(\frac{4 \pi}{3 f_{\rho}}\right)^{\beta} \delta^{-\beta n},
$$

where $A_{v} \approx 0.8, \beta=0.19$, and $f_{\rho}=\rho_{1 / 2} / \rho_{*}$ is the ratio of the half-radius density to the average density $\rho_{*}=3 M / 4 \pi R_{*}^{3}$. In Appendix $\mathrm{D}$, we derive $f_{\rho}$ for some appropriate density profiles for the SN I progenitors we are interested in. Matzner \& McKee (1999) show that the final velocity of the fluid is given by $v_{f}\left(r_{0}\right)=f_{v}\left(r_{0}\right) v_{s}\left(r_{0}\right)$, where $f_{v} \approx 2$.

The thermal diffusion length scale is

$$
D\left(\delta_{m}, t\right) \approx \sqrt{\frac{c t}{3 \kappa \rho\left(\delta_{m}, t\right)}},
$$

where the density is given from continuity to be

$$
\rho=-\frac{M}{4 \pi r^{2} t}\left(\frac{d v_{f}}{d \delta_{m}}\right)^{-1} \approx \frac{1+1 / n}{\beta} \frac{\Delta M}{4 \pi t^{3} v_{f}^{3}} .
$$

The diffusion depth is approximated as the location where $D$ is roughly equal to the thickness of a given shell of material (Piro et al. 2010)

$$
\Delta r \approx \frac{\beta}{1+1 / n} v_{f} t
$$

Setting $\Delta r=D$, we find

$$
\begin{aligned}
\delta_{m}= & {\left[\frac{1+1 / n}{\beta} \frac{f_{v} A_{v} c}{3 \kappa} \frac{4 \pi t^{2}}{M}\left(\frac{E}{M}\right)^{1 / 2}\right.} \\
& \left.\times\left(\frac{4 \pi}{3 f_{\rho}}\right)^{\beta}\left(\frac{3 f_{\rho}}{n+1}\right)^{\beta /(1+1 / n)}\right]^{(1+1 / n) /(1+1 / n+\beta)} .
\end{aligned}
$$

This expression is then used to derive Equation (8). Note that $\Delta M_{\text {diff }}=M \delta_{m} \propto f_{\rho}^{-0.04}$ for $\beta=0.19$ and $n=3$. In Appendix $\mathrm{D}$, we find that $f_{\rho}$ ranges from $\approx 0.01$ for $\mathrm{SN} \mathrm{Ib} / \mathrm{c}$ progenitors (this is what is used for the numerical factor in Equation (8)) to $\approx 1$ for SN Ia progenitors, so the prefactor of Equation (8) varies by $\approx 20 \%$ between these two very different scenarios.

An important issue in estimating the diffusion depth is the opacity $\kappa$, which we consider to be constant here. This approximation is motivated by the detailed opacity study of Pinto \& Eastman (2000), which deserves some discussion. For the heavy elements present in $\mathrm{SNe}$ Ia, the dominant opacity arises from line transitions mainly concentrated in the UV. Nevertheless, on a timescale of $\approx 10$ days post-explosion, they find that the flux mean opacity is roughly constant and compares favorably with the Rosseland mean opacity. This means that semi-analytic and numerical models that assume a simply constant opacity should be sufficient to reproduce the gross properties of the diffusion wave. It should be noted that on the timescales that Pinto \& Eastman (2000) compare these opacities that iron-group elements are playing an important role. Since we find ${ }^{56} \mathrm{Ni}$ at the depths the diffusion wave is probing, it means our models are at least self-consistent in the sense that the opacity we use is appropriate for the conditions we consider. More work is needed though to understand the opacity, and thus thermal diffusion wave depth, in cases where the layers are poor in iron-group elements.

\section{APPENDIX D}

\section{THE DENSITY STRUCTURE FACTOR}

Even though the diffusion depth is relatively insensitive to the dimensionless density structure factor $f_{\rho}$, it is worth approximating just how greatly it varies for different SN I progenitors. Using Matzner \& McKee (1999), for a radiative envelope of constant opacity

$$
f_{\rho}=\frac{\pi}{144} \frac{\beta^{4}}{1-\beta}\left(\frac{\mu m_{p}}{k_{\mathrm{B}}}\right)^{4} G^{3} M^{2} a,
$$

where $1-\beta=L_{*} / L_{\text {Edd }}$ is the ratio of the stellar luminosity to the Eddington limit. For a Thomson opacity with hydrogendeficit material this ratio is

$$
1-\beta=0.51\left(\frac{L}{10^{5} L_{\odot}}\right)\left(\frac{M}{3 M_{\odot}}\right)^{-1} .
$$

This then gives $f_{\rho} \approx 0.015$ for an $\mathrm{SN} \mathrm{Ib} / \mathrm{c}$ progenitor.

For degenerate electrons as in a WD progenitor for $\mathrm{SNe} \mathrm{Ia}$, the equation of state is $P=K \rho^{1+1 / n}$. For the case of nonrelativistic electrons $n=3 / 2$ and $K=9.91 \times 10^{12} \mu_{e}^{-5 / 3}$, and for relativistic electrons $n=3$ and $K=1.23 \times 10^{15} \mu_{e}^{-4 / 3}$, where $\mu_{e}$ is the molecular weight per electron and $K$ is in cgs units. In this case,

$$
f_{\rho}=\frac{4 \pi R_{*}^{3}}{3 M}\left[\frac{G M}{(n+1) K R_{*}}\right]^{n} \approx 1.3\left(\frac{M}{1.4 M_{\odot}}\right)^{2},
$$


where in the last expression we have assumed relativistic electrons and $\mu_{e}=2$.

\section{REFERENCES}

Arnett, W. D. 1979, ApJL, 230, L37

Ben-Ami, S., Gal-Yam, A., Filippenko, A. V., et al. 2012, ApJL, 760, L33

Bloom, J. S., Kasen, D., Shen, K. J., et al. 2012, ApJL, 744, L17

Chevalier, R. A. 1992, ApJ, 394, 599

Colgate, S. A. 1974, ApJ, 187, 333

Corsi, A., Ofek, E. O., Gal-Yam, A., et al. 2012, ApJL, 747, L5

Dessart, L., Hillier, D. J., Li, C., \& Woosley, S. 2012, MNRAS, 424, 2139

Dessart, L., Hillier, D. J., Livne, E., et al. 2011, MNRAS, 414, 2985

Drout, M. R., Soderberg, A. M., Gal-Yam, A., et al. 2011, ApJ, 741, 97

Falk, S. W. 1978, ApJL, 225, L133

Filippenko, A. V., Li, W. D., Treffers, R. R., \& Modjaz, M. 2001, in ASP Conf

Ser. 246, IAU Colloq. 183: Small Telescope Astronomy on Global Scales, ed. B. Paczynski, W.-P. Chen, \& C. Lemme (San Francisco, CA: ASP), 121

Foley, R. J., Challis, P. J., Filippenko, A. V., et al. 2012, ApJ, 744, 38

Gandel'Man, G. M., \& Frank-Kamenetskii, D. A. 1956, SPhD, 1, 223

Hachinger, S., Mazzali, P. A., Sullivan, M., et al. 2013, MNRAS, 429, 2228

Hachisu, I., Matsuda, T., Nomoto, K., \& Shigeyama, T. 1991, ApJL, 368, L27

Iglesias, C. A., \& Rogers, F. J. 1996, ApJ, 464, 943

Joggerst, C. C., Woosley, S. E., \& Heger, A. 2009, ApJ, 693, 1780

Kaiser, N., Aussel, H., Burke, B. E., et al. 2002, Proc. SPIE, 4836, 154

Kasen, D., \& Bildsten, L. 2010, ApJ, 717, 245

Kasen, D., \& Woosley, S. E. 2009, ApJ, 703, 2205

Katz, B., Budnik, R., \& Waxman, E. 2010, ApJ, 716, 781

Katz, B., Sapir, N., \& Waxman, E. 2012, ApJ, 747, 147

Klein, R. I., \& Chevalier, R. A. 1978, ApJL, 223, L109
Kleiser, I. K. W., Poznanski, D., Kasen, D., et al. 2011, MNRAS, 415, 372

Lattimer, J. M., \& Prakash, M. 2001, ApJ, 550, 426

Law, N. M., Kulkarni, S. R., Dekany, R. G., et al. 2009, PASP, 121, 1395

Matzner, C. D., \& McKee, C. F. 1999, ApJ, 510, 379

Modjaz, M., Li, W., Butler, N., et al. 2009, ApJ, 702, 226

Nakar, E., \& Sari, R. 2010, ApJ, 725, 904

Nakar, E., \& Sari, R. 2012, ApJ, 747, 88

Nugent, P. E., Sullivan, M., Cenko, S. B., et al. 2011, Natur, 480, 344

Parrent, J. T., Howell, D. A., Friesen, B., et al. 2012, ApJL, 752, L26

Pinto, P. A., \& Eastman, R. G. 2000, ApJ, 530, 757

Piro, A. L. 2012, ApJ, 759, 83

Piro, A. L., Chang, P., \& Weinberg, N. N. 2010, ApJ, 708, 598

Popov, D. V. 1993, ApJ, 414, 712

Rabinak, I., Livne, E., \& Waxman, E. 2012, ApJ, 757, 35

Rabinak, I., \& Waxman, E. 2011, ApJ, 728, 63

Rau, A., Kulkarni, S. R., Law, N. M., et al. 2009, PASP, 121, 1334

Sakurai, A. 1960, CPAM, 13, 353

Sauer, D. N., Mazzali, P. A., Deng, J., et al. 2006, MNRAS, 369, 1939

Schlegel, D. J., Finkbeiner, D. P., \& Davis, M. 1998, ApJ, 500, 525

Sedov, L. I. (ed.) 1959, Similarity and Dimensional Methods in Mechanics (New York, NY: Academic)

Silverman, J. M., Ganeshalingam, M., Cenko, S. B., et al. 2012, ApJL, 756, L7

Soderberg, A. M., Berger, E., Page, K. L., et al. 2008, Natur, 453, 469

Tanaka, M., Mazzali, P. A., Benetti, S., et al. 2008, ApJ, 677, 448

Taylor, G. 1950, RSPSA, 201, 175

Von Neumann, J. 1947, in Los Alamos Sci. Lab. Tech. Series, Vol. 7, Blast

Waves, ed. L. I. Sedov (Los Alamos, NM)

Woosley, S. E. 1988, ApJ, 330, 218

Woosley, S. E. 2010, ApJL, 719, L204

Woosley, S. E., Langer, N., \& Weaver, T. A. 1995, ApJ, 448, 315

Yoon, S.-C., Langer, N., \& Norman, C. 2006, A\&A, 460, 199

Yoon, S.-C., Woosley, S. E., \& Langer, N. 2010, ApJ, 725, 940 